\title{
Automatic OMR Answer Sheet Evaluation using Efficient \& Reliable OCR System
}

\author{
Dhananjay Kulkarni ${ }^{1}$, Ankit Thakur ${ }^{2}$, Jitendra Kshirsagar ${ }^{3}$, Y. Ravi Raju ${ }^{4}$ \\ Student, Computer Engineering, ARMIET, Asangaon, India ${ }^{1,2,3}$ \\ Asst. Professor, Computer Engineering Department, ARMIET, Asangaon, India ${ }^{4}$
}

\begin{abstract}
In today's modern world of technology when everything is computerized, the Evaluation exercise of examining and assessing the educational system has become absolute necessity. Today, more emphasis is on objective exam which is preferred to analyze scores of the students since it is simple and requires less time in the examining objective answer-sheet as compared to the subjective answer-sheet. This paper proposes a new technique for generating scores of multiple-choice tests which are done by developing a technique that has software based approach with computer \& scanner which is simple, efficient \& reliable to all with minimal cost. Its main benefit to work with all available scanners, In addition no special paper \& colour required for printing for marksheet. To recognize \& allot scores to the answer marked by of the student's Optical character recognition technique is executed here.
\end{abstract}

Keywords: Multiple Choice, analyze, evaluate, Optical Character Recognition.

\section{INTRODUCTION}

In today's global world, the multiple-choice examination is a vital phenomenon of academic system. Multiplechoice test is used in almost all important exam to determine student's academic excellence. Every year thousands of students take such tests in which they answer by highlighting option in OMR sheets. These exams conducting institutions uses a high complex machine for scoring MCQ paper, these machines are very costly and it needs a highly skilled worker who can manage such machine effectively. These institutes deploy costly OMR software along with complex equipment to analyze\& provide scores to the OMR sheets. Thus, small organizations and training institutes cannot use such process of providing scores to such OMR Sheets. They use manual process of grading OMR test responses of a student is time consuming. The importance of our small initiative is that we could build a computer based software that will save precious time by providing simple as well as cost effective solution which is highly reliable \& efficient by having a OCR approach.

The software might do the some of below mentioned activities:

- Examine the OMR answer sheet,

- Detect feedback i.e. response for each question.

- Compare student answer with master key which will be already saved in database.

Thus, our goal is to create a technique that can be executed with computer \& scanner with a software program that will provide scores to these uniquely designed Multiple choice exam with questions having four options for each question \& only one correct answer per question can be chosen by the student. The software will examine to detect the response of each question of the paper by matching with accurate option which is already saved in database. OpenCV will be implemented to facilitate image processing with designed software program for decoding the answer.

\section{PROPOSED SYSTEM}

Various methods of paper correction are available in the education system till date. The most basic method is manual paper correction. In this the system, the MCQ question paper are evaluated manually by the evaluator and then marks are entered in to the system. This process becomes challenging if the number of answer sheets are large. Today lots of competitive exams which are academic, governmental as well as in private sector are conducted. These exams have multiple choice questioning. Thus, a program need to be implemented as a simple software tool.

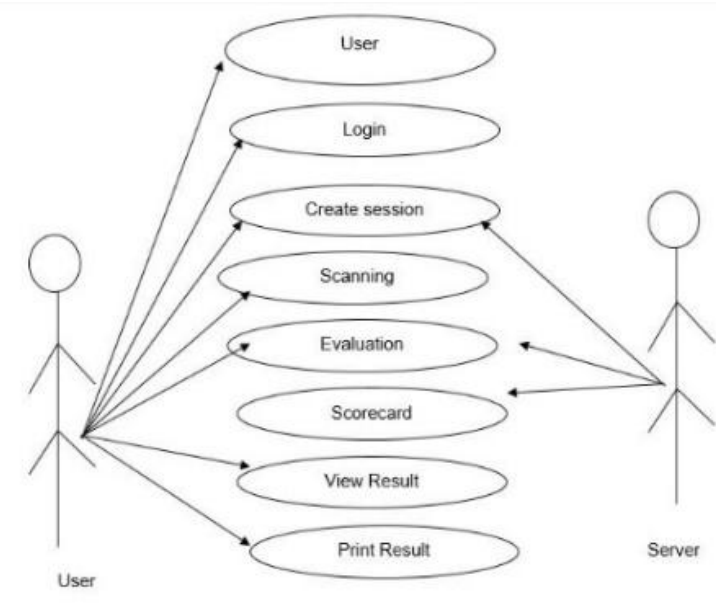


So, OpenCV which is a free open source software application that helps computer programmers for developing software has a simple interface which is systematic\& well-organized technology is used, depending on the scanner used, thousands of OMR sheets per day can be checked with combination of these tools. It contains following main components: software, answer sheets \& scanner. Thus, after completion of exam, the candidate with total selected choices of responses are analyzed\& final scores are generated.

Structured Query Language (SQL)which is a designed as special programming language that is used to create \& manage data efficiently in database management system. Further, Open Source Computer Vision Library (OpenCV) is an open source that is designed with computer vision and machine learning software library. OpenCV tool was created to help a common infrastructure for computer vision applications and also to make notable impact to the use of machine perception in the commercial products. OpenCV makes it easy to utilize and modify the code for various business.

Visual Studio is an integrated development environment (IDE) from Microsoft technologies which is used to create extraordinary computer programs for Microsoft Windows, web sites enhancements, web applications development and other web services. OpenCV (Open Source Computer Vision) IDE free for use under the open-source is as programming function library that is primarily focused at vision for computer which is real-time, this library is cross-platform and which is a multi-pattern program language which has strong typing which is robust, agile as well as indispensable, object oriented programmingwhich is highly functional. It also consists interfaces of $\mathrm{C} \& \mathrm{C}++$, Python, Java and interfaces from MATLAB.

\section{III.COMPONENTS}

\section{A) SCANNER}

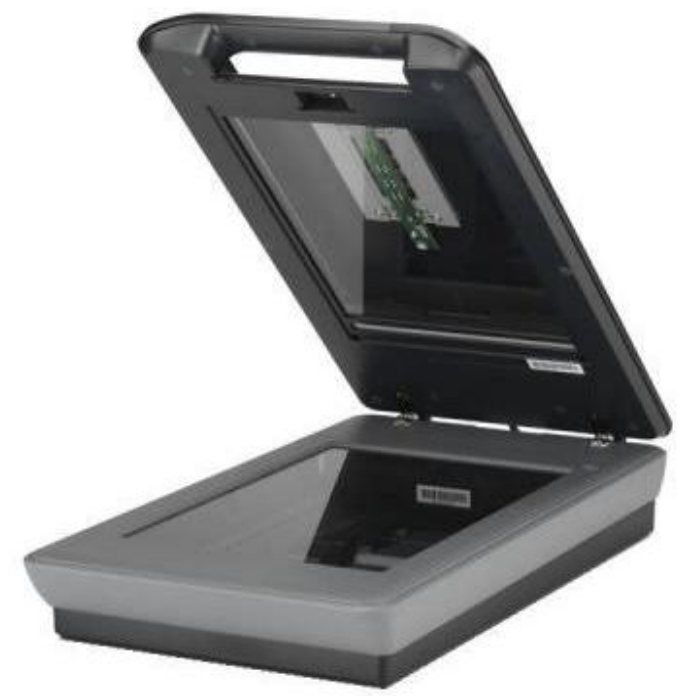

Fig. Scanner

\section{B) ANSWERSHEET}

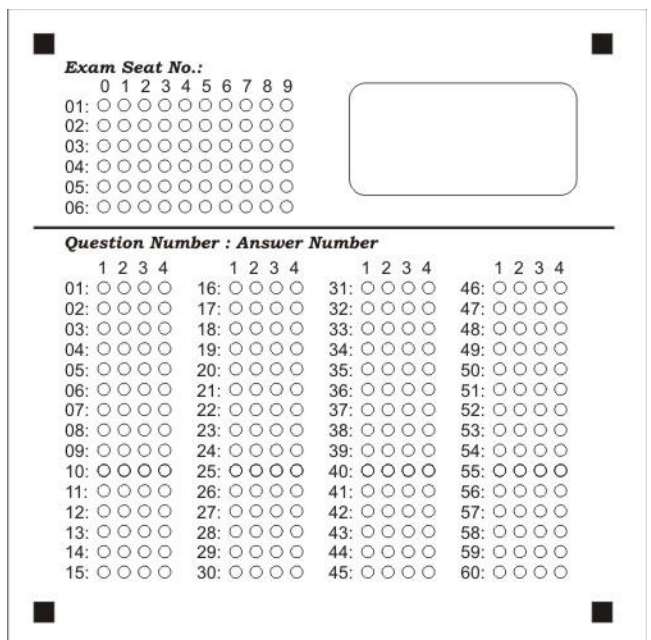

Fig. Format for Answer Sheet

The unique identification section appears on the top part of the answer sheet that consists of customizable template which can be inserted with text boxes for personal information such as Last \& first name, candidate seat no. etc. for identification as it serves as an identity of the candidate undergoing examination.

\section{IV.EASE OF IMPLEMENTATION}

- System requires regular scanner \& space for storage which makes it less expensive.

- Size of file is also less \& is very fast.

- It is simple, agile, efficient $\&$ reliable.

- It can be used with pen etc. for marking the response.

- The answer sheet paper should be printed with $\mathrm{B} / \mathrm{W}$ printer only.

\section{FUTURE SCOPE}

- In a very less time Education institutes can track the academic performance of students which might have chances to be executed in different languages also.

- In Various academic institutes feedback process for staff can also be evaluated.

- We can increase the number of question \& question paper sets in the paper.

- It can be executed at micro level in administration of government \& private sectors for feedback, so that it will enhance future prospects \& endeavours of organization.

\section{VI.CONCLUSION}

Thus, it's a very simple \& low cost proposed system used with scanner to decode answer from OMR test sheets over the traditional expensive OMR (Optical Mark Reader) system.This system is agile, coherent\& has prodigious methodology of evaluation. 


\section{ACKNOWLEDGEMENT}

I would like to express my gratitude\& big thanks to Alamuri Ratnamala Institute of Engineering and Technology for their support\& encouragement at all times.

\section{REFERENCES}

[1] Ramesh, S, (2005). "Exploring the Potential of Multiple Choice Questions in Computer-BasedAssessment of Student Learning". pattern recognition letters, VOL.6,NO.7,PP(199-213).

[2] Bar-Hillel, M., Budescu, D., \&Attali, Y. (2005). Scoring and keying multiple choice tests: A case study in irrationality. Mind and Society, VOL,4,NO.8PP(3-12).

[3] Technical paper: "An Automatic System to Grade Multiple Choice Questions paper based Exams" by Ammar Awny Abbas.

[4] Digital Image Processing (3rd Edition) -Rafael C. Gonzalez.

[5] Learning OpenCV-Computer Vision with them OpenCV LibraryGary Bradski, Adrian Kaehle.

[6] www.wikipedia.com/C\#.net

[7] http://opencv.org 